\title{
DECOMPOSABLE SETS AND MUSIELAK-ORLICZ SPACES OF MULTIFUNCTIONS
}

\author{
ANDRZEJ KASPERSKI \\ Institute of Mathematics, Silesian University of Technology \\ Kaszubska 23, 44-100 Gliwice, Poland \\ E-mail: Andrzej.Kasperski@polsl.pl
}

\begin{abstract}
We introduce the Musielak-Orlicz space of multifunctions $X_{m, \varphi}$ and the set $S_{F}^{\varphi}$ of $\varphi$-integrable selections of $F$. We show that some decomposable sets in Musielak-Orlicz space belong to $X_{m, \varphi}$. We generalize Theorem 3.1 from [6]. Also, we get some theorems on the space $X_{m, \varphi}$ and the set $S_{F}^{\varphi}$.
\end{abstract}

1. Introduction. Decomposability is a basic concept in Multivalued Analysis (see [7], p. 174). A notion of decomposibility has been introduced by Rockafellar in [14]. A similar but different notion has been introduced in [6] and [7] and we will use this notion. The Musielak-Orlicz spaces of multifunctions were introduced and studied in [8]-[11]. The Musielak-Orlicz space of multifunctions $X_{m, \varphi}$ has been introduced in [11]. The aim of this note is to obtain a generalization of Theorem 3.1 from [6] and Theorem 3.8, Chapter 2 from [7]. All definitions and theorems connected with Musielak-Orlicz spaces can be found in [12]. Definitions and theorems connected with multifunctions can be found in [1]-[7], [13] and [14].

Let $(\Omega, \Sigma, \mu)$ be a measure space with a nonnegative, nontrivial $\sigma$-finite and complete measure $\mu$. Let $\varphi$ be a $\varphi$-function, i.e., $\varphi: \Omega \times R \rightarrow R_{+}, \varphi(t, u)$ is an even, continuous function of $u$, equal to zero iff $u=0$ and nondecreasing for $u \geq 0$ for every $t \in \Omega$, is a measurable function of $t \in \Omega$ for every $u \in R$ and $\lim _{u \rightarrow \infty} \varphi(t, u)=\infty$ for $\mu$-a.e. $t \in \Omega$. Moreover, if $\varphi(t, \cdot)$ is a convex function for every $t \in \Omega$, then we shall say that the $\varphi$-function $\varphi$ is convex. Let $L^{\varphi}(\Omega, \Sigma, \mu)$ be the Musielak-Orlicz function space generated by the modular

$$
\rho(x)=\int_{\Omega} \varphi(t, x(t)) d \mu .
$$

2000 Mathematics Subject Classification: 46E30, 46E99, 28B20.

Key words and phrases: separable Banach space, decomposable set, Musielak-Orlicz space of multifunctions, $L^{\varphi}$ selector.

The paper is in final form and no version of it will be published elsewhere. 
Let $\|\cdot\|_{\varphi}^{L}$ denote the Luxemburg norm in $L^{\varphi}(\Omega, \Sigma, \mu)$ if $\varphi$ is convex. Let $Y$ be a real separable Banach space with the norm $\|\cdot\|_{Y}$. Let $\Theta$ denote the zero element of $Y$. If $A, B \subset Y$ are nonempty then we denote

$$
H(A, B)=\max \left(\sup _{x \in A} \inf _{y \in B}\|x-y\|_{Y}, \sup _{y \in B} \inf _{x \in A}\|x-y\|_{Y}\right) .
$$

Denote by $E(Y)$ the set of all nonempty and closed subsets of $Y$. Let

$$
X=\left\{F: \Omega \rightarrow 2^{Y}: F(t) \in E(Y) \text { for every } t \in \Omega\right\} .
$$

Two multifunctions $F, G \in X$ such that $F(t)=G(t)$ for $\mu$-a.e. $t \in \Omega$ will be treated as the same element of $X$.

Now we introduce the function $\mathbf{d}(F, G)$ by the formula:

$$
\mathbf{d}(F, G)(t)=H(F(t), G(t)) \text { for all } F, G \in X \text { and } t \in \Omega .
$$

Let $\mathbf{N}$ be the set of all positive integers. Let $\mathbf{0} \in X$ be such that $\mathbf{0}(t)=\{\Theta\}$ for every $t \in \Omega$. Denote $|F|=\mathbf{d}(F, \mathbf{0})$ for every $F \in X$.

\section{On the space $X_{m, \varphi}$ and the set $S_{F}^{\varphi}$}

Definition 1. We say that $F \in X$ is a step multifunction if

$$
F(t)=\sum_{k=1}^{n} \chi_{A_{k}}(t) B_{k} \text { for every } t \in \Omega
$$

where $\chi_{A}$ is the characteristic function of the set $A, B_{k} \in E(Y)$ for $k=1, \ldots, n, \Omega=$ $\bigcup_{k=1}^{n} A_{k}, A_{k} \in \Sigma$ for $k=1, \ldots, n$ and $A_{i} \cap A_{j}=\emptyset$ for $i \neq j$.

Definition 2. We say that $F \in X$ is measurable if there exists a sequence of step multifunctions $F_{n} \in X$ for every $n \in \mathbf{N}$ such that $\lim _{n \rightarrow \infty} \mathbf{d}\left(F, F_{n}\right)(t)=0$ for $\mu$-a.e. $t \in \Omega$.

Denote:

$$
X_{m}=\{F \in X: F \text { is measurable }\}, \quad X_{m, \varphi}=\left\{F \in X_{m}:|F| \in L^{\varphi}(\Omega, \Sigma, \mu)\right\},
$$

It is easy to see that $\mathbf{d}(F, G) \in L^{\varphi}(\Omega, \Sigma, \mu)$ if $F, G \in X_{m, \varphi}$.

By [7], Chapter 2, Theorem 1.35, if $F \in X_{m}$, then $F$ is measurable and graph measurable in the sense of [7], Chapter 2, Definition 1.1.

The space $X_{m, \varphi}$ will be called the Musielak-Orlicz spaces of multifunctions.

By $L^{\varphi}((\Omega, \Sigma, \mu), Y)$ we will denote the set of all strongly measurable functions $f$ : $\Omega \rightarrow Y$ such that $\|f(\cdot)\|_{Y} \in L^{\varphi}(\Omega, \Sigma, \mu)$.

In [11] the following was proved:

TheOrem 1. Let $F_{n} \in X_{m, \varphi}$ for every $n \in \mathbf{N}$. If for every $\epsilon>0$ and every $a>0$ there exists $K>0$ such that $\int_{\Omega} \varphi\left(t, a \mathbf{d}\left(F_{m}, F_{n}\right)(t)\right) d \mu<\epsilon$ for all $m, n>K$, then there exists $F \in X_{m, \varphi}$ such that $\int_{\Omega} \varphi\left(t, a \mathbf{d}\left(F_{n}, F\right)(t)\right) d \mu \rightarrow 0$ as $n \rightarrow \infty$ for every $a>0$.

COROLlary 1. Let the $\varphi$-function $\varphi$ be convex, then the function

$$
D_{\varphi}(F, G)=\|\mathbf{d}(F, G)\|_{\varphi}^{L}
$$

for all $F, G \in X_{m, \varphi}$ is a metric in $X_{m, \varphi}$, so $\left\langle X_{m, \varphi}, D_{\varphi}>\right.$ is a complete metric space. 
Let $F \in X$. Denote

$$
S_{F}^{\varphi}=\left\{f \in L^{\varphi}((\Omega, \Sigma, \mu), Y): f(t) \in F(t) \mu \text { a.e. }\right\} .
$$

Definition 3. The $\varphi$-function $\varphi$ will be called locally integrable if $\int_{A} \varphi(t, u) d \mu<\infty$ for every $u>0$ and $A \in \Sigma$ with $\mu(A)<\infty$.

Applying the proof of Proposition 3.3, Proposition 2.17 and Remark 3.4 Chapter 2 from [7] we easily obtain the following:

LEMMA 1. Let the $\varphi$-function $\varphi$ be locally integrable and fulfil the condition $\Delta_{2}$, then for every $F \in X_{m}$ such that $S_{F}^{\varphi} \neq \emptyset$ there exists a sequence $\left\{f_{n}\right\} \subset L^{\varphi}((\Omega, \Sigma, \mu), Y)$ such that $F(t)=\overline{\left\{f_{n}\right\}(t)}$ for $\mu$-a.e. $t \in \Omega$.

Corollary 2. Let the $\varphi$-function $\varphi$ be locally integrable and fulfil the condition $\Delta_{2}$. Let $F, G \in X_{m}$ be such that $S_{F}^{\varphi}=S_{G}^{\varphi} \neq \emptyset$, then $F(t)=G(t)$ for $\mu$-a.e. $t \in \Omega$.

LEMMA 2. Let the $\varphi$-function $\varphi$ be locally integrable, convex and fulfil the condition $\Delta_{2}$. Let $F \in X_{m}$ and the sequence $\left\{f_{n}\right\} \subset L^{\varphi}((\Omega, \Sigma, \mu), Y)$ be such that $F(t)=\overline{\left\{f_{n}\right\}(t)}$ for $\mu$-a.e. $t \in \Omega$. Then for every $f \in S_{F}^{\varphi}$, every $a>0$, every $\epsilon>0$, there exists a finite measurable partition $\left\{A_{1}, \ldots, A_{n}\right\}$ of $\Omega$ such that $\int_{\Omega} \varphi\left(t, a\left\|f(t)-\sum_{i=1}^{n} \chi_{A_{i}}(t) f_{i}(t)\right\|_{Y}\right) d \mu<\epsilon$.

Proof. We may assume that $f(t) \in F(t)$ for every $t \in \Omega$. Let $a, \epsilon>0$ be arbitrary. Take a strictly positive $\delta \in L^{1}(\Omega, \Sigma, \mu)$ satisfying $\int_{\Omega} \delta d \mu<\frac{\epsilon}{3}$. Then there exists a countable measurable partition $\left\{B_{i}\right\}$ of $\Omega$ such that

$$
\varphi\left(t, a\left\|f(t)-f_{n}(t)\right\|_{Y}\right)<\delta(t) \text { for every } t \in B_{n} .
$$

Take an integer $n$ such that

$$
\sum_{k=n+1}^{\infty} \int_{B_{k}} \varphi\left(t, 2 a\|f(t)\|_{Y}\right) d \mu<\frac{2}{3} \epsilon, \quad \sum_{k=n+1}^{\infty} \int_{B_{k}} \varphi\left(t, 2 a\left\|f_{1}(t)\right\|_{Y}\right) d \mu<\frac{2}{3} \epsilon
$$

and define a finite measurable partition $\left\{A_{1}, \ldots, A_{n}\right\}$ as follows:

$$
A_{1}=B_{1} \cup\left(\bigcup_{i=n+1}^{\infty} B_{i}\right)
$$

and $A_{j}=B_{j}$ for $j=2, \ldots$ Then we have

$$
\begin{aligned}
\int_{\Omega} \varphi\left(t, a\left\|f(t)-\sum_{m=1}^{n} \chi_{A_{m}}(t) f_{m}(t)\right\|_{Y}\right) d \mu=\sum_{m=1}^{n} \int_{A_{m}} \varphi\left(t, a\left\|f(t)-f_{m}(t)\right\|_{Y}\right) d \mu \\
\quad=\sum_{m=1}^{n} \int_{B_{m}} \varphi\left(t, a\left\|f(t)-f_{m}(t)\right\|_{Y}\right) d \mu+\sum_{m=n+1}^{\infty} \int_{B_{m}} \varphi\left(t, a\left\|f(t)-f_{1}(t)\right\|_{Y}\right) d \mu \\
\quad \leq \int_{\Omega} \delta(t) d \mu+\frac{1}{2} \sum_{k=n+1}^{\infty} \int_{B_{k}} \varphi\left(t, 2 a\|f(t)\|_{Y}\right) d \mu+\frac{1}{2} \sum_{k=n+1}^{\infty} \int_{B_{k}} \varphi\left(t, 2 a\left\|_{1}\right\|_{Y}\right) d \mu<\epsilon .
\end{aligned}
$$

Definition 4. Let $M$ be a set of measurable functions $f: \Omega \rightarrow Y$. We call $M$ decomposable (with respect to $\Sigma$ ) if $f_{1}, f_{2} \in M$ and $A \in \Sigma$ imply $\chi_{A} f_{1}+\chi_{\Omega \backslash A} f_{2} \in M$. 
THEOREM 2. Let the $\varphi$-function $\varphi$ be locally integrable, convex and fulfil the condition $\Delta_{2}$. Let $M$ be a nonempty and closed subset of $L^{\varphi}((\Omega, \Sigma, \mu), Y)$. Then there exists an $F \in X_{m}$ such that $M=S_{F}^{\varphi}$ if and only if $M$ is decomposable.

Proof. It is clear that $S_{F}^{\varphi}$ is necessarily decomposable and closed (with respect to norm) in $L^{\varphi}((\Omega, \Sigma, \mu), Y)$. To prove the converse, let $M$ be a nonempty, closed, decomposable subset of $L^{\varphi}((\Omega, \Sigma, \mu), Y)$. By Lemma 1, there exists a sequence $\left\{f_{n}\right\} \subset L^{\varphi}((\Omega, \Sigma, \mu), Y)$ such that $\left\{f_{n}(t)\right\}$ is dense in $Y$ for each $t \in \Omega$. For each $i \in \mathbf{N}$ and $a>0$, let

$$
r_{i}(a)=\inf \left\{\int_{\Omega} \varphi\left(t, a\left\|f_{i}(t)-g(t)\right\|_{Y}\right) d \mu: g \in M\right\}
$$

and choose a sequence $\left\{g_{i j}\right\} \subset M$ such that

$$
\int_{\Omega} \varphi\left(t, a\left\|f_{i}(t)-g_{i j}(t)\right\|_{Y}\right) d \mu \rightarrow r_{i}(a) .
$$

Define $F \in X_{m}$ by $F(t)=\overline{\left\{g_{i j}(t)\right\}}$. We shall prove $M=S_{F}^{\varphi}$. For each $f \in S_{F}^{\varphi}, \epsilon>0$ and $a>0$, by Lemma 2 we can take a finite measurable partition $\left\{A_{1}, \ldots, A_{n}\right\}$ of $\Omega$ and $\left\{h_{1}, \ldots, h_{n}\right\} \subset\left\{g_{i j}\right\}$ such that

$$
\int_{\Omega} \varphi\left(t, a\left\|f(t)-\sum_{k=1}^{n} \chi_{A_{k}} h_{k}(t)\right\|_{Y}\right) d \mu<\epsilon .
$$

Since $\sum_{k=1}^{n} \chi_{A_{k}} h_{h} \in M$, this implies $f \in M$. Hence $S_{F}^{\varphi} \subset M$. Now suppose $M \neq S_{F}^{\varphi}$. Then there exist an $f \in M$ and $A \in \Sigma$ with $0<\mu(A)<\infty$, and a $\delta>0$ such that

$$
\inf _{i, j}\left\|f(t)-g_{i j}(t)\right\| \geq \delta, \text { for } t \in A .
$$

Take an integer $i$, fixed in the rest of the proof, such that the set

$$
B=A \cap\left\{t \in \Omega:\left\|f(t)-f_{i}(t)\right\|_{Y}<\delta / 3\right\}
$$

has a positive measure, and let

$$
g_{j}^{\prime}=\chi_{B} f+\chi_{\Omega \backslash B} g_{i j}, \quad j \in \mathbf{N} .
$$

Then, since $\left\{g_{j}^{\prime}\right\} \subset M$ and for $t \in B$

$$
\left\|f_{i}(t)-g_{i j}(t)\right\|_{Y} \geq\left\|f(t)-g_{i j}(t)\right\|_{Y}-\left\|f(t)-f_{i}(t)\right\|_{Y}>2 \delta / 3
$$

it follows that for $j \in \mathbf{N}$

$$
\begin{aligned}
\int_{\Omega} \varphi\left(t, a \| f_{i}(t)-g_{i j}\right. & \left.(t) \|_{Y}\right) d \mu-r_{i}(a) \\
& \geq \int_{\Omega} \varphi\left(a\left\|f_{i}(t)-g_{i j}(t)\right\|_{Y}\right) d \mu-\int_{\Omega} \varphi\left(a\left\|f_{i}(t)-g_{j}^{\prime}(t)\right\|_{Y}\right) d \mu \\
& =\int_{B} \varphi\left(t, a\left\|f_{i}(t)-g_{i j}(t)\right\|_{Y}\right) d \mu-\int_{B} \varphi\left(t, a\left\|f_{i}(t)-(t)\right\|_{Y}\right) d \mu \\
& \geq \int_{B}(\varphi(t, 2 a \delta / 3)-\varphi(t, a \delta / 3)) d \mu>0
\end{aligned}
$$

because $\varphi$ is strictly increasing with respect to $u>0$. Letting $j$ go to infinity, we have a contradiction. 
We have for $\varphi(t, u)=u^{p}$ for every $t \in \Omega$, where $1 \leq p<\infty$, Theorem 3.1 from [6].

LEMma 3. Let the $\varphi$-function $\varphi$ be locally integrable, convex and fulfil the condition $\Delta_{2}$. Let $F \in X_{m}$ and $S_{F}^{\varphi} \neq \emptyset$. Then for every $a>0$

$$
\sup \left[\rho\left(a\|f(\cdot)\|_{Y}\right): f \in S_{F}^{\varphi}\right]=\int_{\Omega} \sup \left\{\varphi\left(t, a\|x\|_{Y}\right): x \in F(t)\right\} d \mu .
$$

Proof. Let $a>0$ be fixed. Denote

$$
m^{a}(t)=\sup \left[\varphi\left(t, a\|x\|_{Y}\right): x \in F(t)\right]
$$

for every $t \in \Omega$. It is easy to see that $m^{a}$ is measurable (see Proposition 2.24, Chapter 2 from [7]).

For every $f \in S_{F}^{\varphi}$, $\mu$-a.e. $t \in \Omega$ we have $\varphi\left(t, a\|f(t)\|_{Y}\right) \leq m^{a}(t)$ so

$$
\sup \left[\rho\left(a\|f(\cdot)\|_{Y}\right): f \in S_{F}^{\varphi}\right] \leq \int_{\Omega} m^{a}(t) d \mu .
$$

If $f_{0} \in S_{F}^{\varphi}$ and $\rho\left(a\left\|f_{0}(\cdot)\right\|_{Y}\right)=\infty$ we are done. Thus assume that $\rho\left(a\left\|f_{0}(\cdot)\right\|_{Y}\right)$ is finite. If $\int_{\Omega} m^{a}(t) d \mu=0$, then the proof is evident, so we can assume that $\int_{\Omega} m^{a}(t) d \mu>0$. If $m^{a}(t)=+\infty$ on the set of positive measure the proof is also evident, so we can assume that $m^{a}(t)$ is finite $\mu$-a.e.

Let $\beta<\int_{\Omega} m^{a}(t) d \mu$. We will produce an $f \in S_{F}^{\varphi}$ such that $\beta<\rho\left(a\|f(\cdot)\|_{Y}\right)$ and this will finish the proof. Let $\Omega=\bigcup_{n \in \mathbf{N}} \Omega_{n}$ with $\Omega_{n} \subset \Omega_{n+1}$ and $\mu\left(\Omega_{n}\right)<\infty$ for every $n \in \mathbf{N}$. Also let $\delta: \Omega \rightarrow R_{+} \backslash\{0\}$ be an $L^{1}(\Omega, \Sigma, \mu)$ function. Define $A_{n}=\Omega_{n} \cap\{t \in \Omega$ : $\left.\varphi\left(t, a\left\|f_{0}(t)\right\|_{Y}\right) \leq n\right\}$ and

$$
m_{n}^{a}(t)=\left\{\begin{array}{lll}
m^{a}(t)-\frac{\delta(t)}{n}, & \text { if } \quad t \in A_{n}, m^{a}(t) \leq n, \\
n-\frac{\delta(t)}{n}, & \text { if } \quad t \in A_{n}, m^{a}(t)>n, \\
\varphi\left(t, a\left\|f_{0}(t)\right\|_{Y}\right), & \text { if } \quad t \in \Omega \backslash A_{n}
\end{array}\right.
$$

Evidently $m_{n}^{a} \in L^{1}(\Omega, \Sigma, \mu)$ and $m_{n}^{a} \uparrow m^{a}$ in $\mu$-measure. So passing to a subsequence if necessary, we may assume that $m_{n}^{a}(t) \uparrow m^{a}(t) \mu$-a.e. Thus by the monotone convergence theorem, we deduce that there exists $n_{0} \in \mathbf{N}$ such that $\beta<\int_{\Omega} m_{n_{0}}^{a}(t) d \mu$. Let

$$
G_{a}(t)=F(t) \cap\left\{x \in Y: \varphi\left(t, a\|x\|_{Y}\right) \geq m_{n_{0}}^{a}(t)\right\}
$$

for every $t \in \Omega$. By modifying $G_{a}$ on a $\mu$-null set, we may assume that $G_{a} \neq \emptyset$ for every $t \in \Omega$ and then $G_{a}$ is graph-measurable so (see [7], Chapter 2, Theorems 2.1 and 2.14) there exists $g: \Omega \rightarrow Y$ which is a strongly measurable selection of $G_{a}$. Let

$$
C_{n}=\Omega_{n} \cap\left\{t \in \Omega:\|g(t)\|_{Y} \leq n\right\}
$$

and $f_{n}=\chi_{C_{n}} g+\chi_{\Omega \backslash C_{n}} f_{0}$. It is easy to see that $C_{n} \in \Sigma$. Since $S_{F}^{\varphi}$ is decomposable, we have $f_{n} \in S_{F}^{\varphi}$ and

$$
\begin{aligned}
\rho\left(a\left\|f_{n}(\cdot)\right\|_{Y}\right) & =\int_{C_{n}} \varphi\left(t, a\|g(t)\|_{Y}\right) d \mu+\int_{\Omega \backslash C_{n}} \varphi\left(t, a\left\|f_{0}(t)\right\|_{Y}\right) d \mu \\
& \geq \int_{\Omega} m_{n_{0}}^{a}(t) d \mu+\int_{\Omega \backslash C_{n}}\left[\varphi\left(t, a\left\|f_{0}(t)\right\|_{Y}\right)-m_{n_{0}}^{a}(t)\right] d \mu .
\end{aligned}
$$

Note that $\mu\left(\Omega \backslash C_{n}\right) \rightarrow 0$ and $\int_{\Omega} m_{n_{0}}^{a}(t) d \mu>\beta$, so for some $n_{1} \in \mathbf{N}$ we have

$$
\rho\left(a\left\|f_{n_{1}}(\cdot)\right\|_{Y}\right)>\beta \text {. }
$$


By Theorem 2 and Lemma 3 we obtain the following:

THEOREM 3. Let the $\varphi$-function $\varphi$ be locally integrable, convex and fulfil the condition $\Delta_{2}$. Let $M$ be a nonempty, bounded, decomposable and closed subset of $L^{\varphi}((\Omega, \Sigma, \mu), Y)$. Then there exists an $F \in X_{m, \varphi}$ such that $M=S_{F}^{\varphi}$.

Proof. By Theorem 2, $F \in X_{m}$, by Lemma 3 we have $|F| \in L^{\varphi}(\Omega, \Sigma, \mu)$, so $F \in X_{m, \varphi}$.

COROLlary 3. Let the $\varphi$-function $\varphi$ be locally integrable, convex and fulfil the condition $\Delta_{2}$. Let $M$ be a nonempty, bounded, decomposable and closed subset of $L^{\varphi}((\Omega, \Sigma, \mu), Y)$ and let $M(t)=\{f(t): f \in M\}$ be a closed subset of $Y$ for every $t \in \Omega$. Then there exists an $F \in X_{m, \varphi}$ such that $M(t)=F(t) \mu$-a.e.

Proof. Denote $S_{F}^{\varphi}(t)=\left\{f(t): f \in S_{F}^{\varphi}\right\}$ for every $t \in \Omega$. By Lemma 1 we have $S_{F}^{\varphi}(t) \subset$ $F(t) \subset \overline{S_{F}^{\varphi}(t)} \mu$-a.e. So by the assumptions we have $F(t)=S_{F}^{\varphi}(t) \mu$-a.e.

REMARK 1 . Let the $\varphi$-function $\varphi$ be locally integrable, convex and fulfil the condition $\Delta_{2}$. If $F \in X_{m, \varphi}$, then $S_{F}^{\varphi}$ is a bounded and closed subset of $L^{\varphi}((\Omega, \Sigma, \mu), Y)$.

THEOREM 4. Let the $\varphi$-function $\varphi$ be locally integrable, convex and fulfils the $\Delta_{2}$ condition. Let $F_{1}, F_{2} \in X_{m}$ and $S_{F_{1}}^{\varphi}, S_{F_{2}}^{\varphi} \neq \emptyset$. Let $F(t)=\overline{F_{1}(t)+F_{2}(t)}$ for every $t \in \Omega$, then $S_{F}^{\varphi}=\overline{S_{F_{1}}^{\varphi}+S_{F_{2}}^{\varphi}}$.

Proof. It is easy to see that $F \in X_{m}$, so $S_{F}^{\varphi}$ is closed, hence $\overline{S_{F_{1}}^{\varphi}+S_{F_{2}}^{\varphi}} \subset S_{F}^{\varphi}$. On the other hand by Lemma 1 we may find $\left\{f_{1 n}\right\} \subset S_{F_{1}}^{\varphi}$ and $\left\{f_{2 m}\right\} \subset S_{F_{2}}^{\varphi}$ such that $F_{1}(t)=\overline{\left\{f_{1 n}(t)\right\}}$ and $F_{2}(t)=\overline{\left\{f_{2 m}(t)\right\}} \mu$-a.e. Evidently $F(t)=\overline{\left\{f_{1 n}(t)+f_{2 m}(t)\right\}} \mu$-a.e. By Lemma 2 for $f \in S_{F}^{\varphi}$ and $\epsilon>0$ we can find $\left\{A_{1}, \ldots, A_{I}\right\}$ a finite $\Sigma$-partition of $\Omega$ and positive integers $n_{1}, \ldots, n_{I}, m_{1}, \ldots, m_{I}$ such that

$$
\|\| f(\cdot)-\sum_{k=1}^{I} \chi_{A_{k}}\left(f_{1 n_{k}}(\cdot)+f_{2 m_{k}}(\cdot)\right)\left\|_{Y}\right\|_{\varphi}^{L}<\epsilon .
$$

Hence $f \in \overline{S_{F_{1}}^{\varphi}+S_{F_{2}}^{\varphi}}$, so $S_{F}^{\varphi}=\overline{S_{F_{1}}^{\varphi}+S_{F_{2}}^{\varphi}}$.

For $\varphi(t, u)=u^{p}$ for every $t \in \Omega$, where $1 \leq p<+\infty$, we have Proposition 3.28, Chapter 2 from [7].

3. Final remark. The results of this paper can be extended to the case that the $\varphi$ function $\varphi$ is not convex but only strictly increasing with respect to $u$. Clearly we must then use the $F$-norm in Musielak-Orlicz space.

\section{References}

[1] J. Appell, H. T. Nguyen and P. P. Zabrejko, Multivalued superposition operators in ideal spaces of vector functions. I, II, Indag. Math., N.S. 2 (1991) 385-395, 397-409.

[2] Z. Artstein and J. A. Burns, Integration of compact set-valued functions, Pacific Journal of Math. 58 (1975), 297-307.

[3] J.-P. Aubin and H. Frankowska, Set-Valued Analysis, Birkhäuser, Boston/Basel/Berlin, 1990. 
[4] S. De Blasi and A. Lasota, Daniell's method in the theory of the Aumann-Hukuhara integral of set-valued functions, Lincei Rend. Sc. fis. mat. e nat. 45 (1968), 252-256.

[5] S. De Blasi and A. Lasota, Characterization of the integral of set-valued functions, Lincei Rend. Sc. fis. mat. e nat. 46 (1969), 154-157.

[6] F. Hiai and H. Umegaki, Integrals, conditional expectation, and martingales of multivalued functions, J. Multival. Anal. 7 (1977), 149-182.

[7] S. Hu and N. S. Papageorgiou, Handbook of Multivalued Analysis Vol. 1: Theory, Kluwer Academic Publishers, Dordrecht/Boston/London, 1997.

[8] A. Kasperski, Musielak-Orlicz spaces of multifunctions, convergence and approximation, Commentationes Math. 34 (1994), 99-107.

[9] A. Kasperski, Notes on approximation in Musielak-Orlicz spaces of multifunctions, Commentationes Math. 34 (1994), 109-122.

[10] A. Kasperski, Notes on approximation in the Musielak-Orlicz spaces of vector multifunctions, Commentationes Math. Univ. Carolinae 35 (1994), 81-93.

[11] A. Kasperski, Some approximation problems in Musielak-Orlicz spaces of multifunctions, Demonstratio Math. 37 (2004), 393-406.

[12] J. Musielak, Orlicz Spaces and Modular Spaces, Lecture Notes in Mathematics 1034, Springer, Berlin, 1983.

[13] A. Pliś, Remark on measurable set-valued functions, Bull. Acad. Polon. Sci. Sér. Sci. Math. Astronom. Phys. 9 (1961), 857-859.

[14] R. T. Rockafellar, Integrals which are convex functions, Pacific J. Math. 24 (1968), 525539 . 